Hautarzt 2015 • 66:152-153

DOI 10.1007/s00105-015-3588-y

Online publiziert: 27. Februar 2015

(c) Springer-Verlag Berlin Heidelberg 2015

\author{
P. Elsner ${ }^{1}$ - S.M. John ${ }^{2,3,4} \cdot$ Ch. Skudlik 2,3,4 \\ ${ }^{1}$ Klinik für Hautkrankheiten, Universitätsklinikum Jena, Jena, Deutschland \\ ${ }^{2}$ Institut für interdisziplinäre dermatologische Prävention und Rehabilitation (iDerm) an der Universität \\ Osnabrück, Niedersächsisches Institut für Berufsdermatologie, Standort Universität Osnabrück, \\ Osnabrück, Deutschland \\ ${ }^{3}$ Fachgebiet Dermatologie, Umweltmedizin, Gesundheitstheorie, Universität Osnabrück, \\ Osnabrück, Deutschland \\ ${ }^{4}$ Niedersächsisches Institut für Berufsdermatologie, Universität Osnabrück, Osnabrück, Deutschland
}

\title{
Vom Handekzem zum Hautkrebs
} Neues aus der Berufsdermatologie

Am 09.11.2014 wurde feierlich des Falls der Mauer in Berlin vor 25 Jahren gedacht. Nach dem Mauerfall überstürzten sich damals die Ereignisse, und zum 03.10.1990 wurde die deutsche Einheit vollzogen.

Auch im deutschen Berufskrankheitenrecht vollzieht sich in diesen Tagen mit Verspätung - die deutsche Einheit.

In der ehemaligen DDR (und für einen Übergangszeitraum bis 01.01.1992 im Gebiet der neuen Bundesländer) galt die „Liste der Berufskrankheiten gemäß Anlage zur BK-Verordnung der ehemaligen DDR vom 6. Mai 1981“. Diese sah unter Nr. 90 die Anerkennung von „Bösartigen Neubildungen der Haut und zur Krebsbildung neigenden Hautveränderungen “vor, wobei unter dieser Ziffer nicht nur Hautkrebserkrankungen „durch Ruß, Rohparaffin, Teer, Anthrazen, Pech oder ähnliche Stoffe" (entsprechend der in der Bundesrepublik geltenden Ziffer 5102 der Anlage 1 der Berufskrankheitenverordnung) anerkannt wurden, sondern auch Fälle von Hautkrebs durch berufliche Exposition mit UV-Licht.

Im „Vertrag zwischen der Bundesrepublik Deutschland und der Deutschen Demokratischen Republik über die Herstellung der Einheit Deutschlands (Einigungsvertrag)“ vom 31.08.1990 war in Art. 30 (6) vereinbart worden: „Bei der Fortentwicklung der Berufskrankheitenverordnung ist zu prüfen, inwieweit die bisher in dem in Artikel 3 des Vertrags genannten Gebiet geltenden Regelungen berücksichtigt werden können." Diese Prüfung dauerte fast ein Vierteljahr- hundert und fand bezüglich des beruflichen Hautkrebses ihren Abschluss in der Veröffentlichung des Sachverständigenbeirats „Berufskrankheiten“ beim Bundesministerium für Arbeit und Soziales vom 12.08.2013, in der empfohlen wurde, in die Anlage 1 zur BerufskrankheitenVerordnung folgende neue Berufskrankheit aufzunehmen: „Plattenepithelkarzinome oder multiple aktinische Keratosen der Haut durch natürliche UV-Strahlung“. Nun wurde mit der „Dritten Verordnung zur Änderung der BerufskrankheitenVerordnung" zum 01.01.2015 von der Bundesregierung mit Zustimmung des Bundesrates die Empfehlung des Sachverständigenbeirats im Berufskrankheitenrecht wirksam umgesetzt.

\section{》) „Plattenepithelkarzinome oder multiple aktinische Keratosen der Haut durch natürliche UV-Strahlung" sind als neue Berufskrankheiten anerkannt}

Prof. Diepgen, Heidelberg, stellt in diesem Heft die neue BK 5103 vor. Damit ergibt sich die Möglichkeit der Anerkennung der neuen Berufskrankheit für über 2,5 Mio. Arbeitnehmer, die aufgrund ihrer beruflichen Exposition gegenüber natürlicher UV-Strahlung einem erhöhten Hautkrebsrisiko ausgesetzt sind. Konnten Dermatologen schon bisher mit Einverständnis des Versicherten derartige Hautkrebserkrankungen bei Vorliegen der ar- beitstechnischen Voraussetzungen nach $\$ 9$ Abs. 2 SBG VII melden, so ist diese ärztliche Verdachtsmeldung - unabhängig von einer Einwilligung des Versicherten - nunmehr gesetzliche Pflicht.

Neben der Möglichkeit der Anerkennung als Berufskrankheit mit der Folge der Heilbehandlung und einer evtl. Entschädigung sollte die Einführung der neuen Berufskrankheit jedoch vor allem Anlass und Ansporn sein, die Anstrengungen zur Prävention des Hautkrebses nicht nur im Privatleben, sondern auch an gefährdeten Arbeitsplätzen zu erhöhen. Hier gilt es, in den kommenden Jahren neue Präventionsangebote zu entwickeln, wobei auf den langjährigen Erfahrungen mit dem Hautarztverfahren in der Prävention der BK 5101 aufgebaut werden kann. Dank der in den vergangenen Jahrzehnten sowohl ambulant wie auch stationär ausgebauten Präventionsmaßnahmen ist trotz Zunahme gemeldeter Fälle der Anteil der Versicherten mit schweren oder wiederholt rückfälligen Hauterkrankungen, die zur Tätigkeitsaufgabe zwingen, kontinuierlich zurückgegangen mit der Folge, dass immer weniger Berufskrankheiten anerkannt werden mussten, und die Versicherten dank optimierter Schutzmaßnahmen ihren Beruf weiterführen konnten. Welche Maßnahmen in der individuellen ambulanten und stationären Prävention bei Berufsdermatosen inzwischen möglich und etabliert sind, erläutern Herr Prof. Skudlik, Osnabrück, und Frau Prof. Weisshaar, Heidelberg, in ihrem Beitrag. 
Die Qualitätssicherung im Hautarztverfahren ist eine wichtige ärztliche Aufgabe. Die Ergebnisse und Konsequenzen einer großen Studie dazu stellen Frau Dr. Voß, Dr. Brans und Prof. John, Osnabrück, vor.

Auch bei der neuen Berufskrankheit (BK) „Plattenepithelkarzinome oder multiple aktinische Keratosen der Haut durch natürliche UV-Strahlung " fallen die Plattenepithelkarzinome oder multiple aktinische Keratosen nicht „vom Himmel“, sondern entstehen nach dermatologischer Erfahrung aus einzelnen aktinischen Keratosen auf chronisch lichtgeschädigter Haut über einen längeren Zeitraum. Dieses „Fenster der Prävention“ "gilt es zu nutzen. Dann sollte es gelingen, die Zahl der zu erwartenden BK-Anerkennungen nach einem Übergangszeitraum wieder zu reduzieren. Welche Präventionsmöglichkeiten sich dafür anbieten, erörtern Frau Prof. Bauer, Prof. Beissert und Dr. Knusch$k e$, Dresden.

Über den neuen und alten Aufgaben der Berufsdermatologie beim Hautkrebs und beim Handekzem drohen manchmal seltenere Berufsdermatosen wie Infektionskrankheiten und Tropendermatosen übersehen zu werden. Dass auch die Skabies eine Berufskrankheit sein kann und wie mit Skabiesausbrüchen bei Mitarbeitern und zu Pflegenden in Pflegeeinrichtungen umzugehen ist, berichten Frau Lukács, Frau Dr. Schliemann und Prof. Elsner, Jena.

Häufig anzutreffende Unklarheiten über den Begriff der „,beruflichen Hauterkrankung“ versucht schließlich der Beitrag von Prof. Elsner und Frau Dr. Schliemann, Jena, auszuräumen.

Wir hoffen, dass Sie die Beiträge in diesem Leitthemenheft der Zeitschrift Der Hautarzt anregen, in Praxis und Klinik berufsdermatologisch aktiv zu sein und zu bleiben. Vielleicht erwerben Sie sogar das Zertifikat „Berufsdermatologie“ der Arbeitsgemeinschaft für Berufs- und Umweltdermatologie $(\mathrm{ABD})$ ?

\section{Vet les}

Prof. Dr. Peter Elsner

\section{Swen Malk Johm}

Prof. Dr. Swen Malte John

Chistoph Shmeth

Prof. Dr. Christoph Skudlik

\section{Korrespondenzadressen}

Prof. Dr. P. Elsner

Klinik für Hautkrankheiten

Universitätsklinikum Jena

Erfurter Str. 35, 07743 Jena

elsner@derma-jena.de

\section{Prof. Dr. S.M. John, MD}

Institut für interdisziplinäre dermatologische Prävention und Rehabilitation (iDerm) an der Universität Osnabrück

Niedersächsisches Institut für

Berufsdermatologie

Standort Universität Osnabrück

Sedanstr. 115, 49090 Osnabrück

sjohn@uos.de

Prof. Dr. Ch. Skudlik

Institut für interdisziplinäre dermatologische Prävention und Rehabilitation (iDerm) an der Universität Osnabrück

Niedersächsisches Institut für

Berufsdermatologie

Standort Universität Osnabrück

Sedanstr. 115, 49090 Osnabrück

cskudlik@uos.de

\section{Einhaltung ethischer Richtlinien}

Interessenskonflikt. P. Elsner erhielt Vortragshonorare von Astellas, Galderma, GSK, Jenapharm, Leo, L'Oréal, Meda, Novartis und Pierre Fabre und war als Gutachter für gesetzliche und private Unfallversicherungen tätig.

S.M. John erhielt Vortragshonorare von Almirall, Biogen Idec und Galderma und war als Gutachter für gesetzliche Unfallversicherungen tätig.

C. Skudlik erhielt Vortragshonorare von Biogen Idec, Birken und Galderma und war als Gutachter für gesetzliche und private Unfallversicherungen tätig.
Pleyer, U (Hrsg.)

Entzündliche

Augenerkrankungen

Heidelberg: Springer-Verlag 2014, 529 S., 436 Abb., (ISBN 978-3-642-38419-6), Hardcover, 149.99 EUR

Ein Buch, das Spaß macht! Das ist vielleicht nicht der erste Satz, den man von einer Rezension eines wissenschaftlichen Buches erwartet. Ich bin aber der Meinung, dass gerade das Lesen von Fachbüchern einfach Spaß machen muss, denn nur dann kann man mit Begeisterung das dort Dargestellte wahrnehmen. Und: dieses Buch ist ein perfektes Beispiel dafür, dass aufgrund der didaktisch perfekt aufbereiteten Strukturierung mit vielfältigen Fotos, Abbildungen, einfachen Tabellen, Behandlungsalgorithmen und ein Fachbuch nicht "trocken" sein muss. Besonders lobenswert ist, dass der Aufbau der einzelnen Kapitel so gut gelungen ist, dass sowohl das schnelle Querlesen zum Erfassen von relevanten Praxistipps möglich ist, als auch das gespannt neugierige Lesen, um die wissenschaftlichen Hintergründe zu verstehen.

Ebenfalls sehr lobenswert ist, dass bei Diagnostik und Therapie nicht nur sehr pragmatische Tipps gegeben werden, sondern auch auf ein stufenweises Vorgehen geachtet wird, denn zum Glück bedürfen ja nicht alle Erkrankungen gleich der Maximalversorgung.

Auch wenn ich dieses Buch als Dermatologe inhaltlich sicherlich nicht mit der gleichen tiefgehenden Kritik beurteilen kann wie ein Augenarzt, so ist es doch als Dermatologe eine große Freude, dieses Buch in die Hand zu nehmen. Denn gerade bei den entzündlichen Hauterkrankungen sehen wir oft die entzündliche Beteiligung der Augen, und ein synergistisches therapeutisches Vorgehen ist eindeutig im Sinne des Patienten!

T. Zuberbier, Berlin 